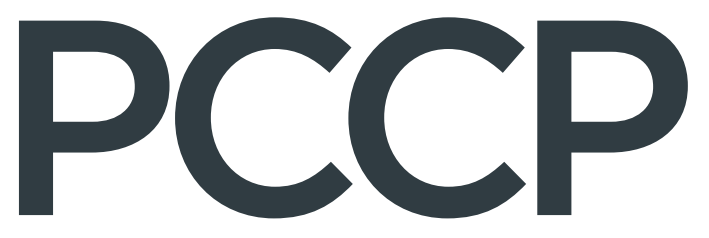

volume 22

Number 32

28 August 2020

Pages 17753-18240

Physical Chemistry Chemical Physics rsc.li/pccp
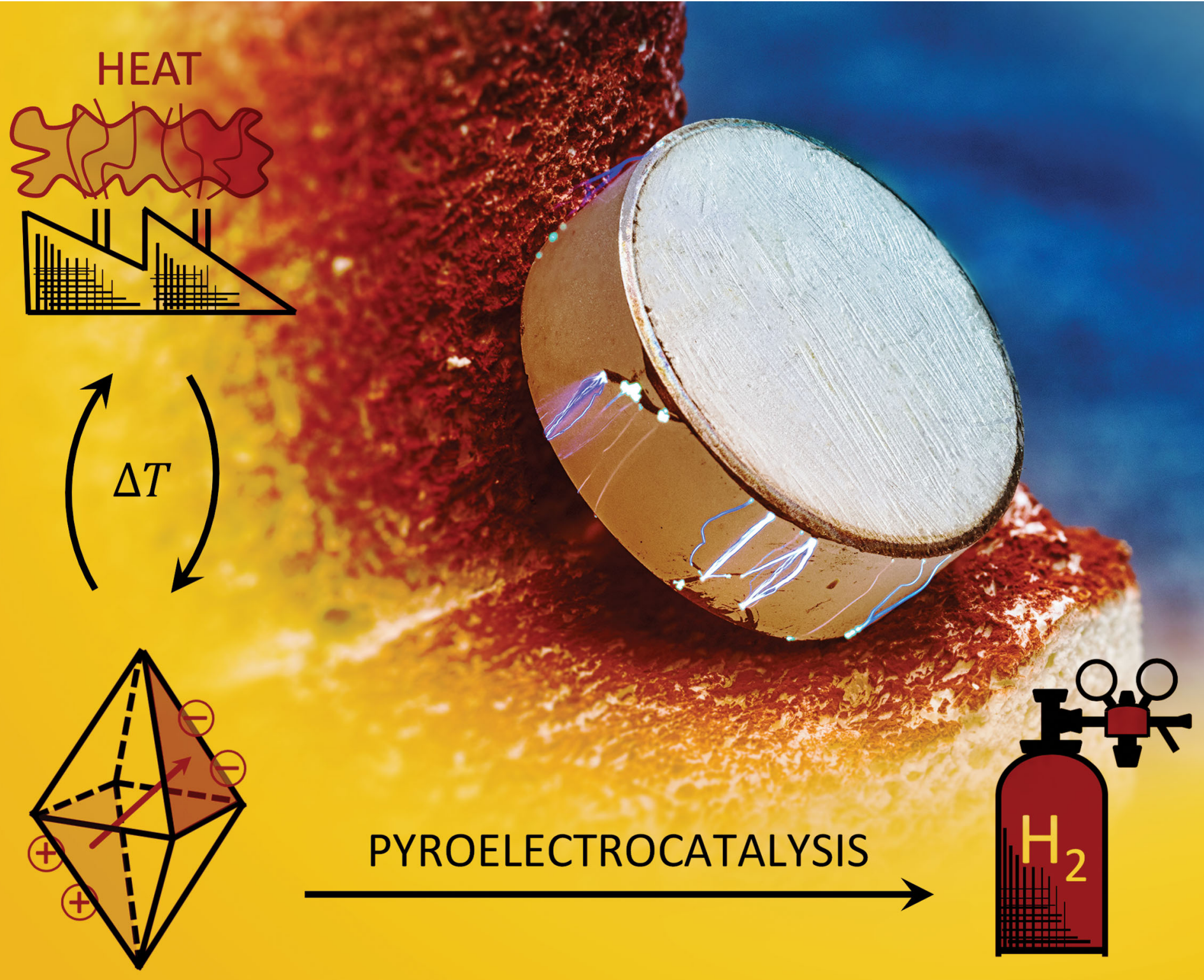

ISSN 1463-9076

ROYAL SOCIETY OF CHEMISTRY

\section{PAPER}

Mateo U. de Vivanco et al.

Pyroelectrically-driven chemical reactions described by a novel thermodynamic cycle 
Check for updates

Cite this: Phys. Chem. Chem. Phys., 2020, 22, 17781

Received 6th March 2020 Accepted 1st April 2020

DOI: $10.1039 / \mathrm{d} 0 \mathrm{cp} 01288 \mathrm{~b}$

rsc.li/pccp

\title{
Pyroelectrically-driven chemical reactions described by a novel thermodynamic cycle $\uparrow$
}

\author{
Mateo U. de Vivanco, (ID $\ddagger^{*^{a}}$ Matthias Zschornak, $\ddagger^{a}$ Hartmut Stöcker, ${ }^{a}$ \\ Sven Jachalke, ${ }^{a}$ Erik Mehner, ${ }^{a}$ Tilmann Leisegang $\left(\mathbb{D}^{\mathrm{ab}}\right.$ and Dirk C. Meyer ${ }^{\mathrm{a}}$
}

Pyroelectrocatalysis is the conversion of thermal energy directly into chemical energy. On the background of renewable energies and the need for efficient industrial processes, the conversion of waste heat into hydrogen is of special relevance. Since the reported thermodynamic cycles for pyroelectric energy harvesting do not fit the conditions encountered in a reactive medium such as water appropriately, we describe a new thermodynamic charge-voltage-cycle characterised by fixed upper and lower potentials. These threshold potentials comprise the redox potential of the reaction of interest - here the hydrogen evolution reaction - as well as an overpotential mainly dictated by the temperature-induced bending of electronic bands in the pyroelectric semiconductor. Because polarisation changes below the threshold are useless for chemical reactions, material properties as well as process conditions have to be chosen accordingly. In particular the particle size along with the temperature difference are shown to determine the conversion efficiency.

\section{Introduction}

Due to the tremendous ecological footprint of fossil fuels and industry, a meaningful measure to slow down environment degradation would be to improve our energy balance by increasing conversion efficiencies and tapping waste energy sources. The reason is that a considerable share of our primary energy (the energy contained in raw materials) is lost during conversion - an estimated $72 \%$ worldwide. ${ }^{1}$ The heat demand of the chemical, metallurgic and manufacturing industries tends to be very high, sometimes achieving process temperatures well above $1000{ }^{\circ} \mathrm{C}$. High-temperature waste heat could be, disregarding some technical obstacles, directly employed to generate electricity e.g. with the aid of the Rankine cycle. ${ }^{2}$

On the other hand, low-grade waste heat usually has temperatures below $100{ }^{\circ} \mathrm{C}$ and its usable extractable work tends to be modest. At the same time, it accounts for the bigger share on rejected energy with approx. $63 \% .^{1}$ Low-grade waste heat

\footnotetext{
${ }^{a}$ Technische Universität Bergakademie Freiberg, Institut für Experimentelle Physik, Leipziger Straße 23, 09596 Freiberg, Germany.

E-mail: mateo.devivanco@physik.tu-freiberg.de; Fax: +49 373139 4314; Tel: +493731393341

${ }^{b}$ Samara State Technical University, Samara Center for Theoretical Materials Science, Molodogvardeyskaya Str. 244, 443100 Samara, Russia

$\dagger$ Electronic supplementary information (ESI) available: Section S1: Olsen and resistive cycles; Section S2: Calculation of the overpotential; Section S3: Calculated pyroelectrochemical yields; Section S4: Pyroelectric coefficients and permittivities; Section S5: Python simulation code. See DOI: 10.1039/d0cp01288b

\$ These authors contributed equally to this work.
}

originates mainly from electricity generation, and to a lower extent from transport and industry branches such as the food and drink industry. ${ }^{3}$ Beside the Rankine cycle and the thermoelectric effect, the pyroelectric effect poses a suitable technology for its recovery. As will be described in this article, water splitting which belongs to the 100 radical innovation breakthroughs for the future - is one possibility for waste heat recovery. ${ }^{4}$

\subsection{The pyroelectric effect}

In its natural state, the crystallographic unit cell of a pyroelectric substance possesses a net dipole moment. Individual dipole contributions sum up throughout the material ${ }^{5}$ resulting in an inner dielectric field $\vec{E}$ and (partial) electric charges at opposite surfaces of the pyroelectric. Under non-vacuum conditions the surface charges attract charges from the surroundings in order to screen the electric field.

The spontaneous polarisation $\vec{P}_{\mathrm{s}}$ of pyroeletric materials is a function of temperature. Upon a temperature increase, either the atoms in the crystal are displaced or the concerted orientations of the dipoles are randomised, depending on the type of material. As a result the dipole moment shrinks and an induced charge imbalance results at the interface to the medium surrounding the pyroelectric.

The polarisation difference $\mathrm{d} \vec{P}$ directly depends on the magnitude of the temperature change $\mathrm{d} T$. The proportionality factor $\vec{p}$ is named the pyroelectric coefficient (eqn (1)), and is a temperaturedependent material property which can be measured. ${ }^{6}$

$$
\mathrm{d} \vec{P}=\vec{p}(T) \mathrm{d} T
$$


Since in most pyroelectric materials there is only one polar axis, the vector $\vec{p}$ may be reduced to a - in most cases negative scalar $p .^{5,7-9}$ The amount of electric charge $Q$ displaced due to $\mathrm{d} T$ is given by eqn (2), where $A$ is the cross-sectional area of the particle perpendicular to the polarisation axis.

$$
\mathrm{d} Q=A \mathrm{~d} P=A p(T) \mathrm{d} T
$$

\subsection{Pyroelectric energy harvesting}

If the pyroelectric material is electrically connected through electrodes, compensation charges will be induced in these (shown in green in Fig. 1a). When the material is heated and its polarisation decreases (Fig. 1b), the excess charge will flow as an electric current (Fig. 1e). Charge neutrality at the new temperature is achieved and the electric flow ceases (Fig. 1c). If the temperature is then lowered, the original dipole moment is restored and current will flow in the opposite direction (Fig. 1d) until charge neutrality prevails again (Fig. 1a).
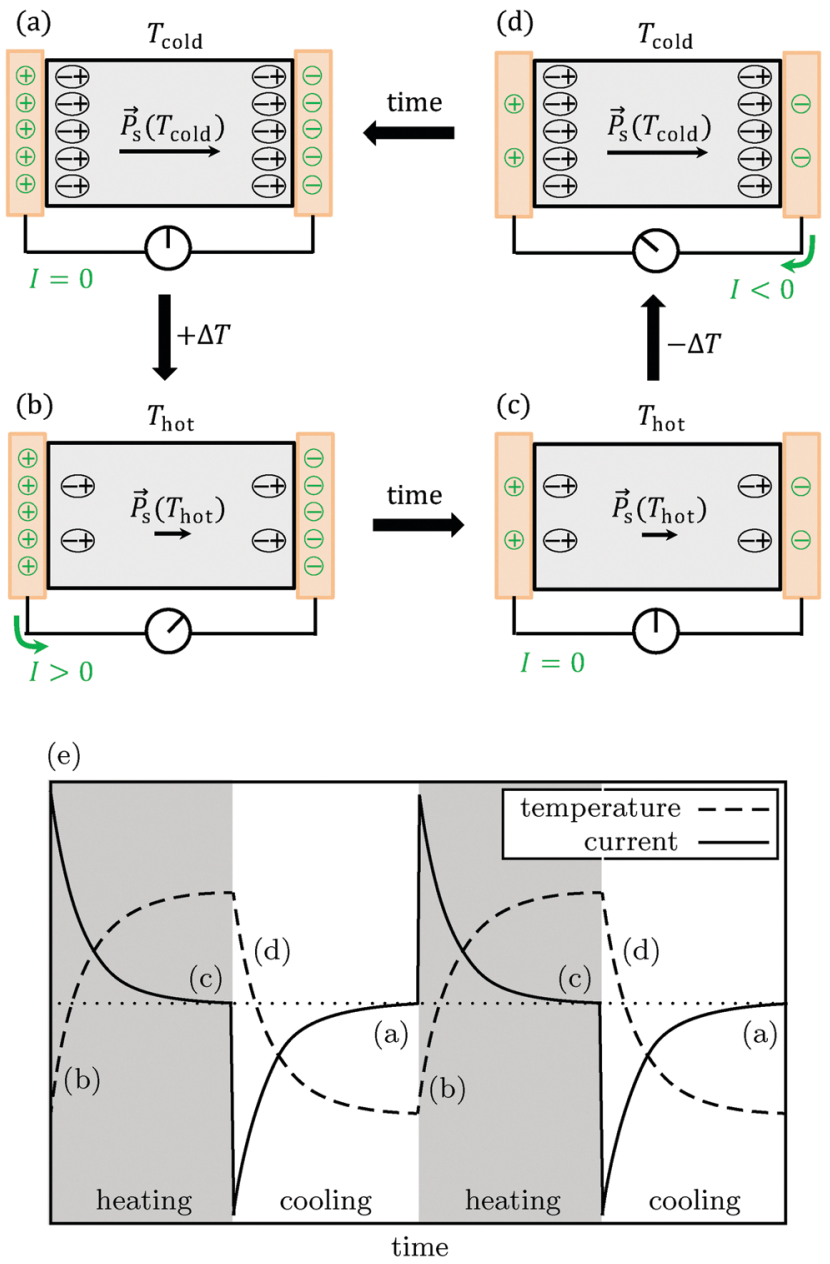

Fig. 1 Electricity generation with the pyroelectric effect. (a), (c) The charge-balanced pyroelectric at thermal equilibrium ( $T_{\text {hot }}>T_{\text {cold }}$ ). The temperature-induced polarisation change upon (b) heating and (d) cooling spurs the flow of charge through an electric circuit. Orange bars represent metal electrodes. (e) Temperature and corresponding pyroelectric current along (a)-(d) in time (two cycles shown).
The described process is the basis for a number of pyroelectric harvesting devices. They differ in the way in which the pyroelectric material is thermally contacted. Leng et al. ${ }^{10}$ switched a pyroelectric between a hot and a cold water stream. With the energy generated they were able to light up LEDs. Lee et al. ${ }^{11}$ pressed the pyroelectric element onto a hot and a cold plate successively. Other authors decided to save the energy required for switching by designing self-moving devices. Such is the example of a bimetal plate which bends to contact the hot or cold plate alternately due to the different thermal expansion coefficients of the two metals. ${ }^{12}$ In addition, a system converting solar and wind energy into temporal temperature differences, as required for pyroelectrics, by use of a light chopper was reported. ${ }^{13}$ The cited articles are examples and by no means a comprehensive list. $\S$

\subsection{Pyroelectrocatalysis}

If the pyroelectric is in contact with a chemically labile medium, redox reactions can be carried out by the unmatched surface charges, as shown in Fig. 2. Starting from the thermally equilibrated material (Fig. 2a), heating diminishes the pyroelectric polarisation (Fig. 2b). The emerging potentials can be capacitively compensated by ions in solution at the beginning. When the potential reaches a certain value or threshold, excess free surface charges $\left(Q_{f}\right)$ can react with the medium or species therein. One end of the crystal becomes oxidising, the other end becomes reducing. Redox reactions can occur on both ends until charge equilibrium is achieved (Fig. 2c). Cooling increases polarisation, the pyroelectric must snatch free charges from its surroundings to balance the polarisation charges (black) caused by the polarisation increase to achieve charge neutrality (Fig. 2d). Redox reactions take place anew, though the reducing and oxidising surfaces reverse.

Such pyroelectrocatalytic reactions have already been reported. The generation of reactive oxygen species was studied in the context of dye oxidation ${ }^{14-16}$ as well as bacteria inactivation. ${ }^{14,17}$ More recently, the feasibility of pyroelectrocatalytic hydrogen production was demonstrated. Kakekhani and Ismail-Beigi ${ }^{18}$ theoretically predicted the formation of hydrogen when cycling a ferroelectric across or underneath its critical temperature: a temperature - and thus potential - change affects the thermodynamic stability of the material surface. As a result, the surface undergoes stabilisation with its surrounding medium through chemisorption; hydrogen emerges as a result (eqn (3)).

$$
\mathrm{H}_{2} \mathrm{O}(\mathrm{l}) \stackrel{\text { pyroel. }, \Delta T}{\longrightarrow} \mathrm{H}_{2}(\mathrm{~g})+\frac{1}{2} \mathrm{O}_{2}(\mathrm{~g}), \quad \Delta H=285.9 \mathrm{~kJ} \mathrm{~mol}^{-1}
$$

Several authors have confirmed the formation of hydrogen experimentally in two set-ups: externally positioned pyroelectrics, which collect pyroelectric current to drive an electrolyser ${ }^{19,20}$ (principle shown in Fig. 1) and internally positioned pyroelectrics, where chemical reactions take place directly on the pyroelectric surface (principle shown in Fig. 2). ${ }^{16}$

$\S$ About 2600 patents are known (google patent search for 'pyroelectr*' and 'harvest*', November 2019). 
(a)

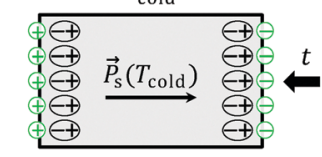

no chemical reaction $+\Delta T$

(b)

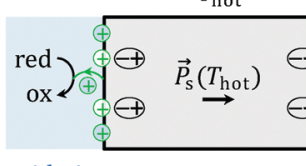

oxidation
$T_{\text {cold }}$ (d)

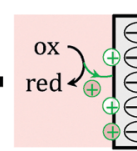

reduction

eduction

(c)

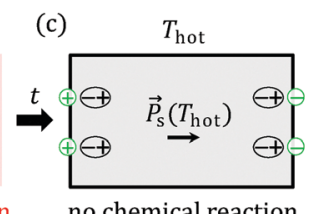

Fig. 2 Simplified model of the pyroelectrocatalytic effect based on externally compensating free (surface) charges (green). (a and c) The charge-balanced pyroelectric at thermal equilibrium in a chemically labile medium. The temperature-induced polarisation change upon (b) heating and (d) cooling generates an electric potential (excess charges are shaded red and blue) able to trigger redox reactions within the medium. ox: oxidised chemical species, red: reduced chemical species. Not included are further screening layers on the surface (e.g. electrical double layer).

The existing theoretical background on pyroelectricity (based on eqn (2)) is straightforward and effective in describing energy harvesting. However, it has major boundaries when it comes to describing chemical reactions. According to eqn (2) even the smallest temperature stimulus would displace charge and enable chemical conversions. Kakekhani and Ismail-Beigi ${ }^{18}$ already pointed out the necessity of a minimum variation of chemical potential and thus critical temperature window in order to realise pyroelectrocatalysis.

\subsection{Thermodynamic pyroelectric cycles}

For the pyroelectric effect to occur, at least one heat source and one heat sink are required for temperature cycling. The pyroelectric process thus resembles a heat engine and is as such subject to the second law of thermodynamics.

The thermodynamic cycle for pyroelectrics bearing the highest energy density was developed by Olsen $e t ~ a l .{ }^{21}$ (also refer to Bowen et al. ${ }^{22}$ ). It is a four-step process which, in electric analogy to the heat engine, produces work from variations of polarisation (charge) in an external electric field (voltage). A schematic of the cycle can be found in Fig. S1a (ESI $\dagger$ ). Lefeuvre $e t a l .{ }^{23}$ and Sebald et $a .^{24}$ described the synchronised electric charge extraction, short SECE, consisting of four partial processes of varying the temperature to charge the pyroelectric and subsequently releasing the charge.

Although these cycles are veracious descriptions of the pyroelectric process, they do not represent the situation of chemical energy harvesting i.e. pyroelectrocatalysis well. When e.g. very small pyroelectric particles should harvest chemical energy, connecting the pyroelectric to a resistor is impossible or undesired. The reactive medium would close the circuit through ion migration. In the simplest setup, no external field would be applied, leaving us with the resistor cycle (Fig. S1b, ESI †). Since the work performed in a charge-voltage diagram depends on the actual path taken, a new cycle describing the exact path must be defined. Most recently, Schlechtweg et al. ${ }^{25}$ proposed a timedependent model to predict hydrogen yields from pyroelectrocatalysis. They describe the dynamics of pyroelectrocatalysis by modelling the process electrically as an RC-circuit with faradaic processes. Up to now it appears to be the best model describing pyroelectrocatalysis, albeit it neither accounts for material specifics nor does it regard interface properties in detail. Thus, the accuracy of their predictions is limited.

The present paper deals with formal aspects of pyroelectrocatalytic energy harvesting. We develop a physical model to explain the occurrence of chemical reactions on pyroelectric surfaces. Furthermore we propose an appropriate thermodynamic cycle, i.e. one that does not require external electric fields and where work is a function of the real pyroelectric potential in a chemically labile medium. Estimations of the efficiency of the cycle are presented. In addition we study the influence of various operational and material parameters on the chemical output and compare simulation results to real experiments reported in literature.

\section{The pyroelectrocatalytic threshold cycle (PTC)}

\subsection{General characteristics}

The main discordance between existing thermodynamic cycles for electric energy harvesting and the process for the harvesting of chemical energy is that the potential of a pyroelectric particle in a reactive medium (e.g. water) can be raised only up to a certain limit or threshold. The varying temperature induces polarisation changes which again induce surface-bound charges. These charges increase the electrostatic potential and can become operative at the interface to the surrounding fluid after a certain energetic barrier - the activation energy - is overcome.

Let us call the voltage difference required for chemical reactions to take place the threshold voltage, $V_{\text {th }}$. Charge build-up from zero to this threshold reaction potential is not available for chemical reactions (high electric resistance of the medium, capacitive regime). Once $V_{\text {th }}$ has been reached, the next unit charge activated by an infinitesimal temperature increase $\left(T_{n-1}<T_{n}\right)$ will not increment the surface potential further, because it will have the appropriate energy for becoming operative within the fluid causing a chemical reaction (drop of the ohmic resistance of the medium, selective faradaic regime).

In particular, the cycle describes the electrochemical energy exchange for pyroelectrics with surfaces that are stable within the parameter regime of operation. From an electronic point of view, the density of states of the pyroelectric is inert with respect to the accumulated threshold voltage, so that apart from physisorption effects redox reactions will take place at the surface. In that way, this model is more general than that presented by Kakekhani and Ismail-Beigi ${ }^{18}$ for catalytic surfaces.

\subsection{Cycle description}

We postulate the thermodynamic cycle shown in Fig. 3, in which the pyroelectrocatalytic work is displayed as an area in a 


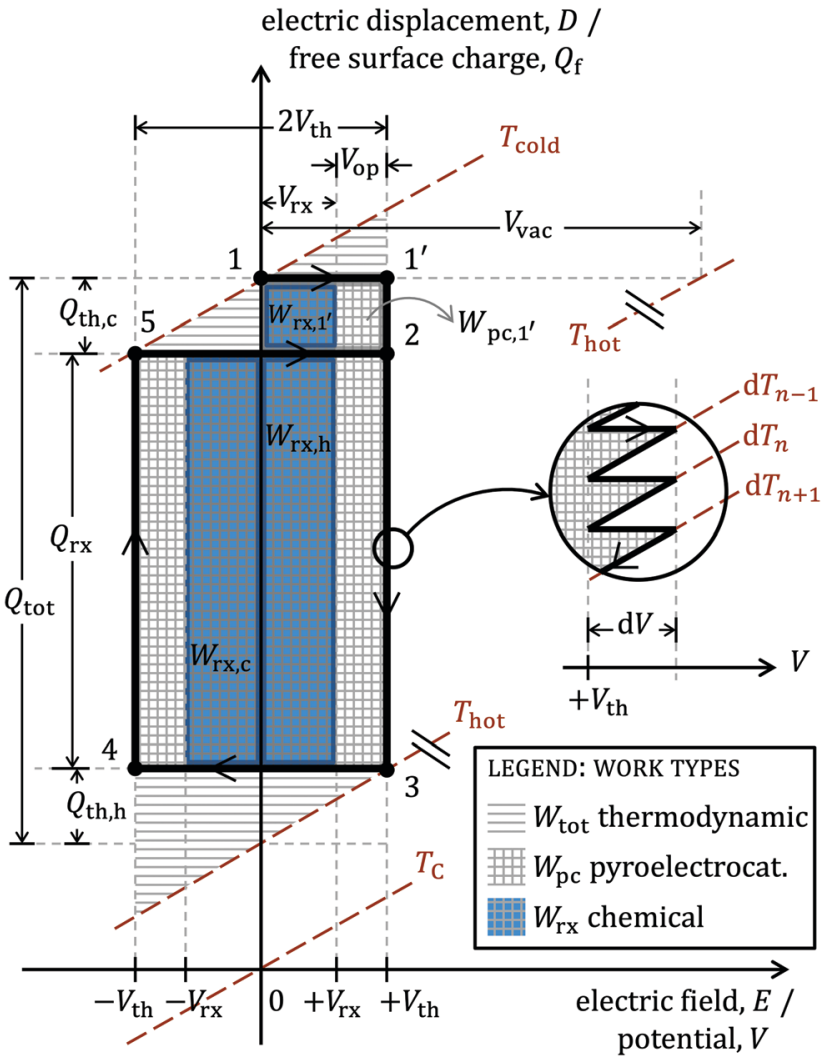

Fig. 3 Pyroelectrocatalytic threshold cycle (PTC) of a pyroelectric particle in direct contact with a reactive medium (assuming infinite resistance of the medium underneath the threshold potential $\left.V_{\mathrm{th}}\right)$. This is a modified thermal cycle, operated between a low $\left(T_{\text {cold }}\right)$ and high $\left(T_{\text {hot }}\right)$ termperature, in which the shadowed areas are proportional to the total thermodynamic work $W_{\text {tot, }}$ the chequered areas represent the pyroelectrocatalytically extractable work $W_{p c}$ and the blue areas represent the work that is stored as chemical energy in the form of reaction products $\left(\mathrm{H}_{2}\right)$. Note that the chemical work is contained in the pyroelectrocatalytic work, which is in turn contained in the (pyro)electric/thermodynamic work. Isotherms are shown as dashed red lines. $T_{\mathrm{C}}$ : Curie temperature. $Q_{\text {tot: }}$ : total displaced charge, $Q_{\mathrm{th}, \mathrm{c} / \mathrm{h}}$ : charge invested in setting the threshold at 'c' cold/' $\mathrm{h}$ ' hot temperature regime, $Q_{\mathrm{rx}}$ : charge effectively causing chemical reaction. $V_{\text {op }}$ : overpotential, $V_{r x}$ : potential effectively used for reaction, $V_{\text {vac }}$ : maximum potential reachable in vacuum. The zoom displays the supposed course of the potential at $+V_{\mathrm{th}}$. $\mathrm{dV}$ : voltage change caused by infinitesimal temperature change $d T_{n}$.

$D E$-diagram (equivalent to a $Q V$-diagram). The dielectric displacement $D$ relates to the electric field $E$ and the polarisation $P$ (eqn (4)), whereby $P$ is caused by bound charges (material inner dipoles), $D$ is caused by free charges (attached external charges) and $E$ is the resultant physical field considering both internal and external charge. $\varepsilon_{0}$ represents the vacuum permittivity. Isotherms are drawn as diagonal lines, since $D$ increases with $E$. The isotherm crossing through the origin corresponds to the Curie temperature $T_{\mathrm{C}}$, above which the spontaneous polarisation is lost.

$$
\vec{D}=\varepsilon_{0} \vec{E}+\vec{P}
$$

The pyroelectrocatalytic threshold cycle (PTC) can be read as follows $(\uparrow:$ rise $/ \downarrow$ : decrease $/=$ : constant):
- 1: $E=0$. A pyroelectric at the low temperature $T_{\text {cold }}$ has a certain polarisation $P_{1}$ and is electrically compensated by free surface charges $D_{1}=-P_{1}$.

- $1 \rightarrow 1^{\prime}: T \uparrow, P \downarrow, E \uparrow, D=$. A charge imbalance in the order of $Q_{\text {th,c }}$ (threshold charge at low temperature, c stands for 'cold') is thermally induced in the form of surface-bound charge by the polarisation decrease without charge transfer. The potential difference rises to $+V_{\text {th }}$. The field increase matches the polarisation decrease and $D$ remains constant at $D_{1}$.

- $1^{\prime} \rightarrow 2: T \uparrow, P \downarrow, E=, D \downarrow$. The charge imbalance induces electrolysis. The electric potential is maintained constant, as the shrinking $P_{\mathrm{s}}$ is compensated by the reacting charges. The electric potential moves along a sawtooth-type path at $+V_{\mathrm{th}}$, as shown in the zoom inset of Fig. 3. The magnitude of $\mathrm{d} V$ is estimated to be: $\mathrm{d} V=\mathrm{d} Q / C=e /\left(\varepsilon_{0} \varepsilon_{\mathrm{r}} a\right) \approx 10^{-19} /\left(10^{-11} \times 10^{2} \times\right.$ $10^{-6}$ ) $=10^{-4} \mathrm{~V}$ for particle sizes $a$ in the $\mu \mathrm{m}$-range (refer to eqn (6)). A charge equal to $Q_{\mathrm{th}, \mathrm{c}}$ is transferred with the fluid. The amount of free surface charge $Q_{\mathrm{f}}$ on the pyroelectric is reduced and so is the electric displacement.

- $2 \rightarrow 3: T \uparrow, P \downarrow, E=, D \downarrow$. The temperature increase further induces charge imbalance and electrolysis. The charge $Q_{\mathrm{rx}}$ ('rx' stands for 'reaction') is exchanged with the medium, reducing the amount of free surface charge of the pyroelectric and thus the electric displacement down to $D_{3}$ at the highest temperature $T_{\text {hot }}\left(<T_{\mathrm{C}}\right)$. The electric field $E$ and potential difference $+V_{\text {th }}$ remain constant.

- $3 \rightarrow 4: T \downarrow, P \uparrow, E \downarrow, D=, Q_{\mathrm{f}}=$. Cooling begins. The initial increase in polarisation cancels out the charge imbalance through surface-bound charges (intersect with $D$-axis). Then the pyroelectric surface potential is reversed to $-V_{\mathrm{th}}$. The charge $Q_{\text {th,h }}$ (threshold charge at high temperature) is required for pole reversal. No chemical reactions occur (the free surface charge $Q_{\mathrm{f}}$ remains constant).

- $4 \rightarrow 5: T \downarrow, P \uparrow, E=, D \uparrow$. Charge imbalance induces electrolysis. The charge $Q_{\mathrm{rx}}$ is transferred on the way to $T_{\text {cold }}$. The crystal sides where oxidation and reduction occur are reversed in comparison to the heating process $(2 \rightarrow 3)$. The displacement increases from $D_{3}$ to $D_{2}$.

- $5 \rightarrow 2: T \uparrow, P \downarrow, E \uparrow, D=, Q_{\mathrm{f}}=$. Heating restarts. The decrease in polarisation reverses the charge imbalance caused by surfacebound charges by $Q_{\text {th,c. }}$. The pyroelectric surface potential is reversed from $-V_{\text {th }}$ to $+V_{\text {th }}$ at constant displacement $D_{2}$. No chemical reactions occur.

Note that the path $5 \rightarrow 2$ is not equivalent to the two-step path first along the isotherm $T_{\text {cold }}$ and then at constant potential $+V_{\text {th }}$ over $1^{\prime}$ to 2 . Even if both pathways require the charge $Q_{\mathrm{th}, \mathrm{c}}$, work is a function of the actual path. As a consequence, the PTC (path $5 \rightarrow 2$ ) loses the thermodynamic work contained in the triangle under the isotherm (whose area will be approximately equivalent to the rectangle area $\left.Q_{\mathrm{th}, \mathrm{c}} \times 2 V_{\mathrm{th}}\right)$.

\subsection{Work calculation}

The following derivation is based on cuboid pyroelectric particles of edge length $a$, which can be regarded as small parallel-plate capacitors. Eqn (5) shows the capacitance $C$, in which the plate area is $A=a^{2}$ and the plate separation $d$ is the particle thickness $a$. 
Eqn (6), derived from eqn (2) and the capacitor equation, is used to calculate the potential $V$ on the pyroelectric surface.

$$
\begin{gathered}
C=\varepsilon_{0} \varepsilon_{\mathrm{r}} \frac{A}{d}=\varepsilon_{0} \varepsilon_{\mathrm{r}} a \\
\mathrm{~d} V=\frac{\mathrm{d} Q}{C}=\frac{a p(T) \mathrm{d} T}{\varepsilon_{0} \varepsilon_{\mathrm{r}}(T)}
\end{gathered}
$$

Fig. 4 plots the time courses of relevant parameters. We assume $T_{\text {hot }}$ remains below $T_{\mathrm{C}}$, so that no external field is required for repoling. In this aspect, the PTC resembles the resistive cycle (ESI $\dagger$ ). According to eqn (2), the displaced charge $Q$ follows the same course as the temperature and the generated current is proportional to its first time derivative $(\mathrm{d} Q / \mathrm{d} t)$. In vacuum, the voltage follows the same course as the temperature, reaching $V_{\text {vac }}$ and 0 . However, in aqueous media it will reach $V_{\text {th }}$ to remain constant afterwards due to the ongoing charge transfer during electrolysis. An electrochemical reaction (charge $Q_{\mathrm{rx}}$ flowing into or from the medium) occurs only after

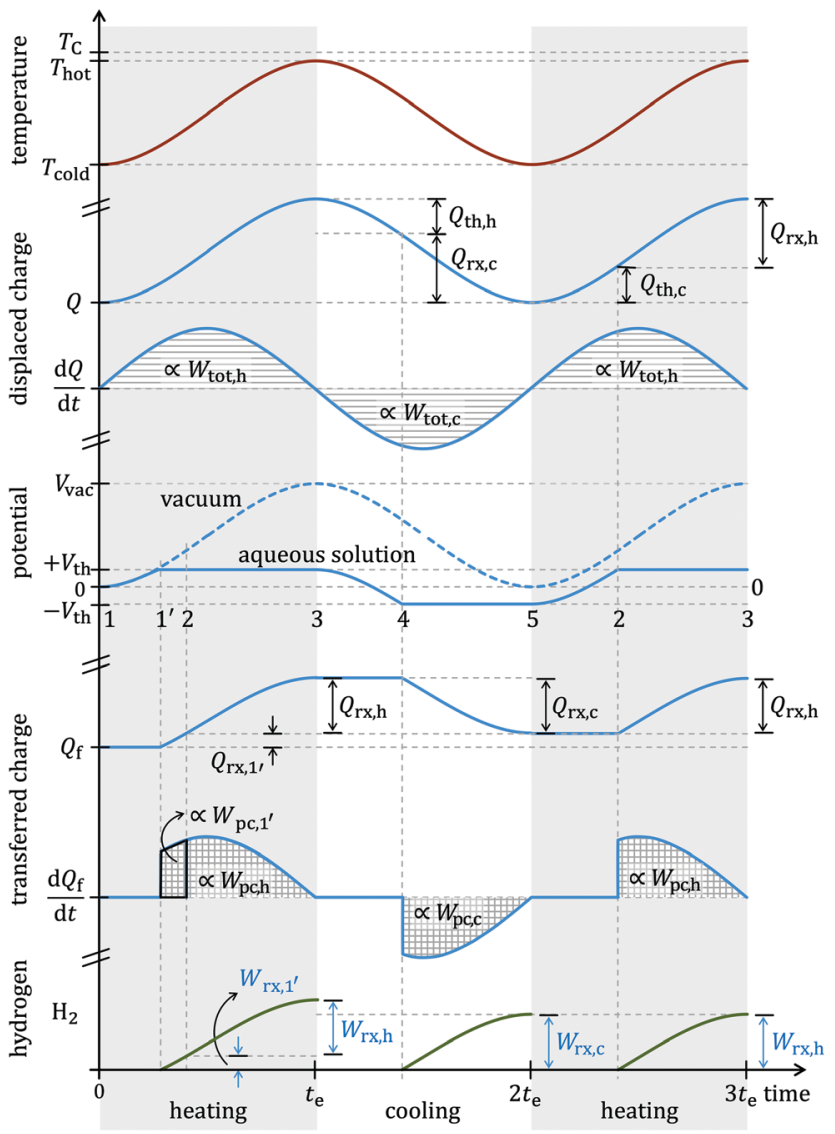

Fig. 4 Time courses of temperature excitation (sinusoidal profile), displaced polarisation charge $Q$, polarisation current $d Q / d t$, surface potential $V$, free surface charge $Q_{\mathrm{f}}$, electrolytic current into the medium $d Q_{\mathrm{f}} / \mathrm{d} t=$ $Q_{r x}$ and moles of hydrogen $\mathrm{H}_{2} \propto W_{r x}$ (cumulative) formed on a pyroelectric particle. Schematic representation. Digits 1-5 correspond to the cycle stages in Fig. 3. Starting from the potential, the time windows lost to the creation of the threshold $\left(Q_{\mathrm{th}, \mathrm{c} / \mathrm{h}}\right)$ and those leveraged to produce chemical work $\left(Q_{r x, c / h}\right)$ can be seen on the $x$-axis (time). Areas of $d Q / d t$ represent the work enclosed in Fig. 3.
$V_{\text {th }}$ has been reached with a rate $\mathrm{d} Q_{\mathrm{rx}} / \mathrm{d} t$ proportional to $\mathrm{d} Q / \mathrm{d} t$. Starting from $0 \mathrm{~V}$, it takes less time to reach $V_{\text {th }}$ than starting at $-V_{\mathrm{th}}$, so that reactions set in earlier on the first run than in all consecutive cycles (note the extra $W_{1^{\prime}}$ produced on the first run, processes $1 \rightarrow 1^{\prime} \rightarrow 2$ in Fig. 3, as well as the extra effective charge $Q_{\mathrm{rx}, 1^{\prime}}$ in Fig. 4, which is lost to the threshold in subsequent runs).

The pyroelectric material performing the PTC delivers electric work for the whole duration of the temperature change. Owing to the fact that the voltage remains pinned at $V_{\text {th }}$ during charge transfer, the work can be calculated with eqn (7). The total thermodynamic work $W_{\text {tot }}$ released by the displacement of bound charges with a total integrated charge of $2 Q_{\text {tot }}(=$ twice the charge per event) according to eqn (2) will be proportional to the total area enclosed between the isotherms and the threshold voltage $\left[-V_{\mathrm{th}},+V_{\mathrm{th}}\right]$ in Fig. 3 (eqn (8)). If $p$ is nearly constant in the working temperature window, then the charge at the hot end $Q_{\text {tot,h }}$ (and thus the work $W_{\mathrm{h}}$ ) will be as large as that at the cold end $Q_{\text {tot,c }}$ (and $W_{\mathrm{c}}$ ). The pyroelectrocatalytic work $W_{\mathrm{pc}}$ (eqn (9)), chequered area, results from the actual course of the state of the pyroelectric within the $D E$-diagram. It has lost the 'triangular' areas merely used to create the threshold potential. Only a fraction of this pyroelectrocatalytic work is chemically stored in the form of reaction products $W_{\mathrm{rx}}$ (eqn (10)), represented by the blue areas. We need to estimate $Q_{\mathrm{rx}}$ and $V_{\mathrm{th}}$ and analyse the factors which further reduce the extractable work when chemical energy - as opposed to total electric work - is targeted.

$$
\begin{gathered}
W=Q V \\
W_{\text {tot }}=W_{\mathrm{h}}+W_{\mathrm{c}}=Q_{\mathrm{tot}, \mathrm{h}} V_{\mathrm{th}}+Q_{\mathrm{tot}, \mathrm{c}} V_{\mathrm{th}}=2 Q_{\mathrm{tot}} V_{\mathrm{th}} \\
W_{\mathrm{pc}}=2 Q_{\mathrm{rx}} V_{\mathrm{th}} \\
W_{\mathrm{rx}}=2 Q_{\mathrm{rx}} V_{\mathrm{rx}}
\end{gathered}
$$

\subsection{Efficiency factors}

2.4.1 Threshold factor $\boldsymbol{\eta}_{\mathbf{t h}}$. The PTC loses a fraction of the enclosed area by creating the threshold potential. When one event - heating or cooling - comes to an end and the next event begins, the existing positive potential $+V_{\text {th }}$ must first be brought back to zero and then beyond to a negative potential $-V_{\text {th }}$. This is the same potential as before, but on the opposite side of the crystal. It is analogous to the process of discharging and recharging a capacitor after reversing the current direction. Only after reaching its final potential $-V_{\text {th }}$ will it be able to trigger electrolysis again. The net change in potential is $2 V_{\text {th }}$ and is found after every temperature switching, twice per cycle. The net charge displaced to achieve this is $Q_{\mathrm{th}, \mathrm{c}}$ at the cold end and $Q_{\mathrm{th}, \mathrm{h}}$ at the hot end. If $p$ is nearly constant, both of these quantities will be equal.

For the estimation of the threshold loss factor, corresponding to a loss of polarisation current of the pyroelectric, we can first calculate the total charge $Q_{\text {tot }}$ displaced per particle during one event - a half cycle - by integrating eqn (2) and second subtract the fraction used for generating the threshold potentials $Q_{\text {th,h/c }}$ (eqn (11)). The displaced charge is given by eqn (12). For narrow temperature ranges, in which $p$ is nearly constant, 
the result of integration can be simplified with the average pyroelectric coefficient as $\bar{p} \cdot \Delta T$.

$$
\begin{aligned}
& Q_{\mathrm{tot}}=Q_{\mathrm{th}, \mathrm{h}}+Q_{\mathrm{rx}, \mathrm{h}}=Q_{\mathrm{th}, \mathrm{c}}+Q_{\mathrm{rx}, \mathrm{c}} \\
& Q_{\mathrm{tot}, \mathrm{h}}=\int_{T_{\mathrm{cold}}}^{T_{\mathrm{hot}}} A p(T) \mathrm{d} T \approx a^{2} \bar{p} \Delta T
\end{aligned}
$$

To determine $Q_{\mathrm{th}, \mathrm{h} / \mathrm{c}}$, we need to relate charge and potential (capacitance). Displacing the charge $Q_{\mathrm{th}, \mathrm{h}}$ or $Q_{\mathrm{th}, \mathrm{c}}$ generates a potential difference of $2 V_{\text {th }}$ at each particle (eqn (13)). Similarly, the total charge displaced for one event generates the maximum potential difference $V_{\text {vac }}$ in a non-reactive medium such as vacuum (eqn (14)). $\bar{\varepsilon}_{\mathrm{r}}$ is the temperature averaged relative permittivity.

$$
\begin{gathered}
Q_{\mathrm{th}}=Q_{\mathrm{th}, \mathrm{c} / \mathrm{h}}=2 V_{\mathrm{th}} C=2 V_{\mathrm{th}} \varepsilon_{0} \varepsilon_{\mathrm{r}}\left(T_{\mathrm{c} / \mathrm{h}}\right) a \\
Q_{\mathrm{tot}}=V_{\mathrm{vac}} C=V_{\mathrm{vac}} \varepsilon_{0} \bar{\varepsilon}_{\mathrm{r}} a
\end{gathered}
$$

The charge loss associated with the generation of the threshold can now be expressed through the efficiency factor $\eta_{\text {th }}$ (eqn (15)), which represents the vertical area loss between the isotherms at $T_{\text {cold }}$ and $T_{\text {hot }}$ in Fig. 3. $\eta_{\text {th }}$ depends on $p$ and $\varepsilon$, as well as on the particle size $a$ and $\Delta T$. As a consequence, for a too small particle size, $\Delta T$ or $p$ the whole cycle duration would be dead time and no reactions would occur. The same effect would be observed for a large $\varepsilon_{\mathrm{r}}$.

$$
\eta_{\mathrm{th}}=\frac{Q_{\mathrm{tot}}-Q_{\mathrm{th}}}{Q_{\mathrm{tot}}}=1-\frac{Q_{\mathrm{th}}}{Q_{\mathrm{tot}}}=1-\frac{2 V_{\mathrm{th}}}{V_{\mathrm{vac}}}=1-\frac{2 V_{\mathrm{th}} \varepsilon_{0} \bar{\varepsilon}_{\mathrm{r}}}{a \bar{p} \Delta T}
$$

(a)

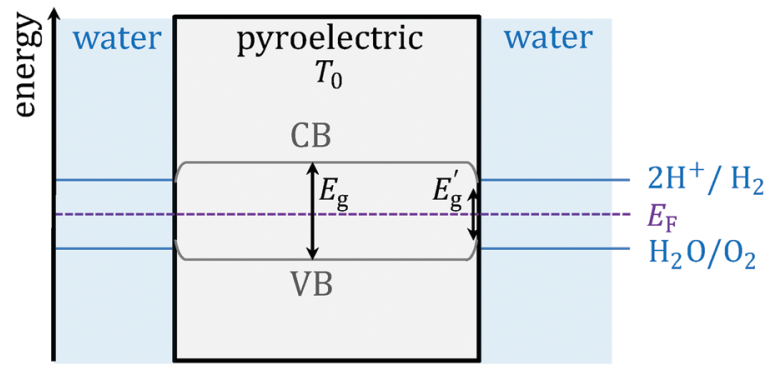

(b)

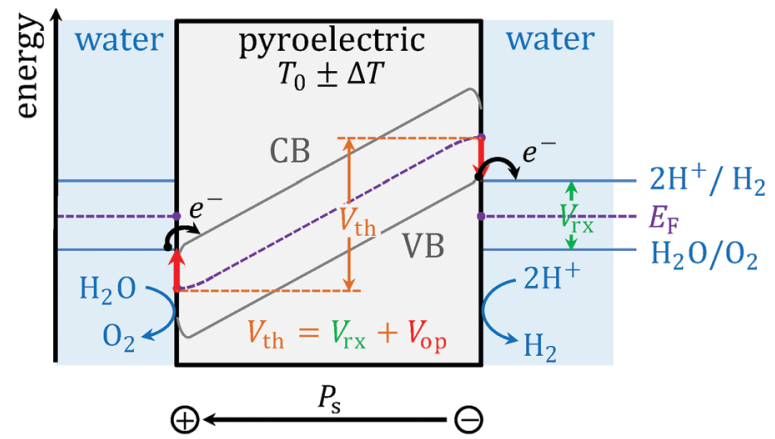

Fig. 5 Electronic states of a pyroelectric particle and water. (a) In thermal equilibrium. (b) Thermal alteration leading to hydrogen (and oxygen) production. Excess $\mathrm{H}_{3} \mathrm{O}^{+}$and $\mathrm{OH}^{-}$produced as by-products may recombine back to water and do not disturb the further pyroelectrocatalytic reaction. For visual clarity in the surface region of the pyroelectric material, we omitted the quadratic bending behaviour at the interfaces in opposite concavity to the depicted band gap decrease.
2.4.2 Overpotential factor $\boldsymbol{\eta}_{\mathbf{o p}}$. The threshold potential $V_{\text {th }}$ required for reaction is comprised of two parts: the usable potential $V_{\mathrm{rx}}$, i.e. the energy being stored in chemical bonds, and the overpotential $V_{\text {op }}$ which is lost as heat (eqn (16) and Fig. 5b).

$$
V_{\mathrm{th}}=V_{\mathrm{rx}}+V_{\mathrm{op}}
$$

2.4.2.1 Band bending $\left(V_{\mathrm{rx}}\right)$. Because all pyroelectrics are piezoelectrics, we can adapt the band model by Starr and Wang ${ }^{26}$ to pyroelectrocatalysis (Fig. 5). At constant temperature and field, the conduction band (CB) and the valence band (VB) of the pyroelectric have the same energy throughout the bulk of the crystal. Since common pyroelectrics have a large band gap $E_{\mathrm{g}}$, the CB will be completely empty while the VB will be full. When the temperature changes, the bands tilt due to the displaced charge and polarisation change (Fig. 5b). In order to produce electrolysis and allow for electronic charge transfer with the fluid, the electrons, which can only be provided by the VB, must be raised in energy above the lowest unoccupied molecular orbital (LUMO) of $\mathrm{H}^{+}$. At the opposite surface, the holes - only available in the $\mathrm{CB}$ - must be lowered in energy below the highest occupied molecular orbital (HOMO) of water. Then the $\mathrm{VB}$ becomes a source of reducing electrons, whilst the CB acts as electron acceptor. The difference between HOMO and LUMO corresponds to the reaction of interest, here water splitting, which requires a minimum potential difference of $V_{\mathrm{rx}}=1.23 \mathrm{~V}$ ( $=E^{\circ}=-\Delta G^{\circ} / n F$, where $G^{\circ}$ is the standard Gibbs free energy, $n$ is the number of transferred electrons and $F$ is the Faraday constant). This potential difference needs to be provided by the heated/cooled pyroelectric. The continuous electronic charge transfer with the fluid maintains a constant imbalance of free surface charges on the pyroelectric, fixing the band tilt and pinning the potential at $V_{\mathrm{th}}$, even though changes in polarisation proceed.

2.4.2.2 Band gap and entropy $\left(V_{\mathrm{op}}\right)$. However, water will not react at $V_{\mathrm{rx}}$ due to missing appropriate reactive sites on the surface. An overpotential is required to compensate this phenomenon. (Other causes for the overpotential such as electric resistance of the aqueous medium, concentration gradients, ${ }^{27}$ bubble formation, etc. are of lesser significance since the current density in a PTC system is orders of magnitude smaller than in a typical electrolysis setup.) Since the electrons can only be transferred into the $\mathrm{CB}$ and out of the $\mathrm{VB}$, an additional band tilt that corresponds to the band gap is needed (red arrows in Fig. 5b). However, due to surface phenomena, adding the full $E_{\mathrm{g}}$ would result in overestimation. Lattice defects and surface stabilisation phenomena such as atomic relaxations and reconstruction commonly create near-surface electronic states at the interface to the fluid reducing the local energy gap to $E_{\mathrm{g}}{ }^{\prime}$ (Fig. 5a). ${ }^{28,29}$ Such reduction depends on the local density of surface states and thus on the specific surface of the pyroelectric. The local density of states can even span the full range of $E_{\mathrm{g}}\left(E_{\mathrm{g}}{ }^{\prime}=0\right)$, making the surface metallic. For the simulation, we have taken several values within the range $\left[0, E_{\mathrm{g}}\right]$. In the specific case of a metallic surface, the reaction still requires a minimum overpotential to at least compensate 
for the entropy increase (eqn (3)). The thermoneutral voltage $V_{\mathrm{n}}=-\Delta H / n F=1.48 \mathrm{~V}$, obtained by replacing $H$, the enthalpy, for $G$ (see ESI $\dagger$ ), ${ }^{30}$ accounts for this.

To sum up, $V_{\mathrm{n}}$ is taken only for the case $E_{\mathrm{g}}{ }^{\prime}=0$ (eqn (17)). The overpotential efficiency term $\eta_{\text {op }}$ - the horizontal component of area loss - is given by eqn (18).

$$
\begin{gathered}
V_{\mathrm{op}}= \begin{cases}E_{\mathrm{g}}{ }^{\prime}, & \text { if } E_{\mathrm{g}}{ }^{\prime} \geq V_{\mathrm{n}}-V_{\mathrm{rx}} \\
V_{\mathrm{n}}-V_{\mathrm{rx}}, & \text { if } E_{\mathrm{g}}{ }^{\prime}<V_{\mathrm{n}}-V_{\mathrm{rx}}\end{cases} \\
\eta_{\mathrm{op}}=\frac{V_{\mathrm{rx}}}{V_{\mathrm{th}}}=\frac{V_{\mathrm{rx}}}{V_{\mathrm{rx}}+V_{\mathrm{op}}}
\end{gathered}
$$

2.4.3 Faradaic factor $\eta_{\mathbf{F}}$. With $\eta_{\text {th }}$ and $\eta_{\text {op }}$ we have the tools to calculate $W_{\mathrm{rx}}$. Still not every single transferred pyroelectric charge has to flow into the desired reaction. An additional efficiency term, the faradaic factor $\eta_{\mathrm{F}}$, may arise for side-reactions. Possible side reactions are here the reoxidation of hydrogen to water at the cathodic particle side and recombination of charge carriers. To avoid the latter, authors report the use of methanol as a hole scavenger. The yields with and without methanol can vary about two orders of magnitude. ${ }^{31}$ So while the blue areas in Fig. 3 represent the maximum possible chemical work, the actual effective chemical work may be lower.

\subsection{Cycle efficiency}

The product of the three efficiency factors $\eta_{\text {th }}, \eta_{\mathrm{op}}$ and $\eta_{\mathrm{F}}$, named here $\eta_{\text {chem }}$ (eqn (19)), characterises the loss in the conversion of (pyro)electric to chemical energy in the form of hydrogen $W_{\mathrm{H}_{2}}$.

$$
\begin{gathered}
W_{\mathrm{H}_{2}}=W_{\mathrm{rx}} \eta_{\mathrm{F}}=2 Q_{\mathrm{rx}} V_{\mathrm{rx}} \eta_{\mathrm{F}}=2 Q_{\mathrm{tot}} V_{\mathrm{th}} \underbrace{\eta_{\mathrm{th}} \eta_{\mathrm{op}} \eta_{\mathrm{F}}}_{\eta_{\mathrm{chem}}} \\
=2 Q_{\mathrm{tot}} V_{\mathrm{th}} \underbrace{\left(\frac{Q_{\mathrm{tot}}-Q_{\mathrm{th}}}{Q_{\mathrm{tot}}}\right)}_{\eta_{\mathrm{th}}} \underbrace{\left.\frac{V_{\mathrm{rx}}}{V_{\mathrm{th}}}\right)}_{\eta_{\mathrm{op}}} \eta_{\mathrm{F}} \\
=2 V_{\mathrm{rx}} \eta_{\mathrm{F}} \underbrace{\left(a^{2} \bar{p} \Delta T\right.}_{Q_{\mathrm{tot}}}-\underbrace{\left.2 V_{\mathrm{th}} \varepsilon_{0} \bar{\varepsilon}_{\mathrm{r}} a\right)}_{Q_{\mathrm{th}}}
\end{gathered}
$$

The next step is to calculate the efficiency of the thermal-tochemical conversion. For this purpose, the net extractable chemical work $W_{\mathrm{H}_{2}}$ is multiplied by the number of particles $N_{\text {particles }}$ and divided by the energy input $q_{\text {in }}$, which is the thermal energy required to heat the pyroelectric. It is assumed that cooling the pyroelectric back to $T_{\text {cold }}$ requires no additional energy input. $N_{\text {particles }}$ is given by the total volume of pyroelectric divided by the particle volume. With a material density $\rho$ and total employed mass $m, N_{\text {particles }}$ is given by eqn (22)

$$
\begin{gathered}
N_{\text {particles }}=\frac{V_{\text {material }}}{V_{\text {particle }}}=\frac{m}{\rho a^{3}} \\
q_{\text {in }}=\int m c_{\mathrm{P}}(T) \mathrm{d} T \approx m \bar{c}_{\mathrm{P}} \Delta T
\end{gathered}
$$

Eqn (23) approximates the specific heat capacity $c_{\mathrm{P}}$ as a constant $\bar{c}_{\mathrm{P}}$ in the temperature window. The final efficiency of the heat-to-hydrogen conversion is shown in eqn (24).

$$
\eta_{\mathrm{H}_{2}}=\frac{N_{\text {particles }} W_{\mathrm{H}_{2}}}{q_{\text {in }}}=\frac{2 \eta_{\mathrm{F}} V_{\mathrm{rx}}}{\rho \bar{c}_{\mathrm{P}}}\left(\frac{\bar{p}}{a}-\frac{2 V_{\mathrm{th}} \varepsilon_{0} \bar{\varepsilon}_{\mathrm{r}}}{a^{2} \Delta T}\right)
$$

The study of eqn (24) reveals which parameters can improve the PTC hydrogen yield. The minuend relates to the total charge displaced pyroelectrically $Q_{\text {tot }}$, whilst the subtrahend is associated to the threshold loss $Q_{\mathrm{th}}$. If the subtrahend is equal to the minuend, $\eta_{\mathrm{H}_{2}}$ becomes zero. That gives rise to a critical particle size (eqn (25)) below which no chemical reactions can take place. In that case, the small surface charges would be merely compensated by an electrochemical double-layer.

$$
a_{\text {crit }}=\frac{2 V_{\mathrm{th}} \varepsilon_{0} \bar{\varepsilon}_{\mathrm{r}}}{\bar{p} \Delta T}
$$

Above $a_{\text {crit }}$, the minuend in eqn (24) is greater than the subtrahend and the course of the efficiency goes up. By taking the partial derivative of $\eta_{\mathrm{H}_{2}}$ (eqn (26)), the optimum particle size $a_{\mathrm{opt}}-$ yielding the highest efficiency - can be determined (eqn (27)). For values of $a$ higher than $a_{\text {opt }}$, the efficiency decreases due to a reduction of the active area for chemical reactions. Table 1 shows $a_{\mathrm{opt}}$ for some common pyroelectrics.

\begin{tabular}{|c|c|c|c|c|c|}
\hline Material & $-\bar{p}\left(\mathrm{C} \mathrm{m}^{-2} \mathrm{~K}^{-1}\right)$ & $\bar{\varepsilon}_{\mathrm{r}}$ & $E_{\mathrm{g}}(\mathrm{eV})$ & $V_{\text {th }}(\mathrm{V})$ & $a_{\mathrm{opt}}(\mathrm{m})$ \\
\hline $\mathrm{BaTiO}_{3}^{32}$ & $1.74 \times 10^{-4}$ & 186 & $3.08^{33}$ & $E_{\mathrm{g}} / 2$ & $1.2 \times 10^{-6}$ \\
\hline $\mathrm{LiTaO}_{3}{ }^{34}$ & $1.80 \times 10^{-4}$ & 56 & $4.9^{35}$ & $E_{\mathrm{g}} / 2$ & $5.4 \times 10^{-7}$ \\
\hline $\mathrm{LiNbO}_{3}{ }^{36}$ & $8.20 \times 10^{-5}$ & 30.3 & 4.7 & $E_{\mathrm{g}} / 2$ & $6.2 \times 10^{-7}$ \\
\hline $\mathrm{Sr}_{0.5} \mathrm{Ba}_{0.5} \mathrm{Nb}_{2} \mathrm{O}_{6}{ }^{37}$ & $6 \times 10^{-4}$ & 400 & $3.28^{38}$ & $E_{\mathrm{g}} / 2$ & $7.7 \times 10^{-7}$ \\
\hline PZT G-1306 (Gulton) ${ }^{36}$ & $4.60 \times 10^{-4}$ & 1900 & $3.25^{39}$ & $E_{\mathrm{g}} / 2$ & $4.8 \times 10^{-6}$ \\
\hline $\mathrm{PVDF}^{40}$ & $2.20 \times 10^{-5}$ & 8 & - & $V_{\mathrm{n}}$ & $3.8 \times 10^{-7}$ \\
\hline PMN-0.26PT ${ }^{41}$ & $1.80 \times 10^{-3}$ & 643 & - & $V_{\mathrm{n}}$ & $3.7 \times 10^{-7}$ \\
\hline
\end{tabular}

$$
\begin{gathered}
\frac{\partial \eta_{\mathrm{H}_{2}}}{\partial a}=\frac{2 \eta_{\mathrm{F}} V_{\mathrm{rx}}}{\rho \bar{c}_{\mathrm{P}}}\left(-\frac{\bar{p}}{a_{\mathrm{opt}}{ }^{2}}+\frac{4 V_{\mathrm{th}} \varepsilon_{0} \bar{\varepsilon}_{\mathrm{r}}}{a_{\mathrm{opt}}{ }^{3} \Delta T}\right) \stackrel{!}{=} 0 \\
a_{\mathrm{opt}}=\frac{4 V_{\mathrm{th}} \varepsilon_{0} \bar{\varepsilon}_{\mathrm{r}}}{\bar{p} \Delta T}=2 a_{\mathrm{crit}}
\end{gathered}
$$

We can also obtain an expression for the maximum efficiency (eqn (28)) by inserting $a_{\text {opt }}$ in eqn (24). Clearly, for maximum efficiency $p$ must be maximised and $\varepsilon_{\mathrm{r}}$ minimised. Unfortunately, both $p$ and $\varepsilon_{\mathrm{r}}$ are mostly not decoupled from one

Table 1 Optimum particle size for common pyroelectric materials, assuming $V_{\text {th }}$ to be $E_{\mathrm{g}} / 2$ or $V_{\mathrm{n}}$. From eqn $(27)$ for $\Delta T=50 \mathrm{~K}\left(25-75^{\circ} \mathrm{C}\right)$ 
(a) $\eta_{\mathrm{H}_{2}}$

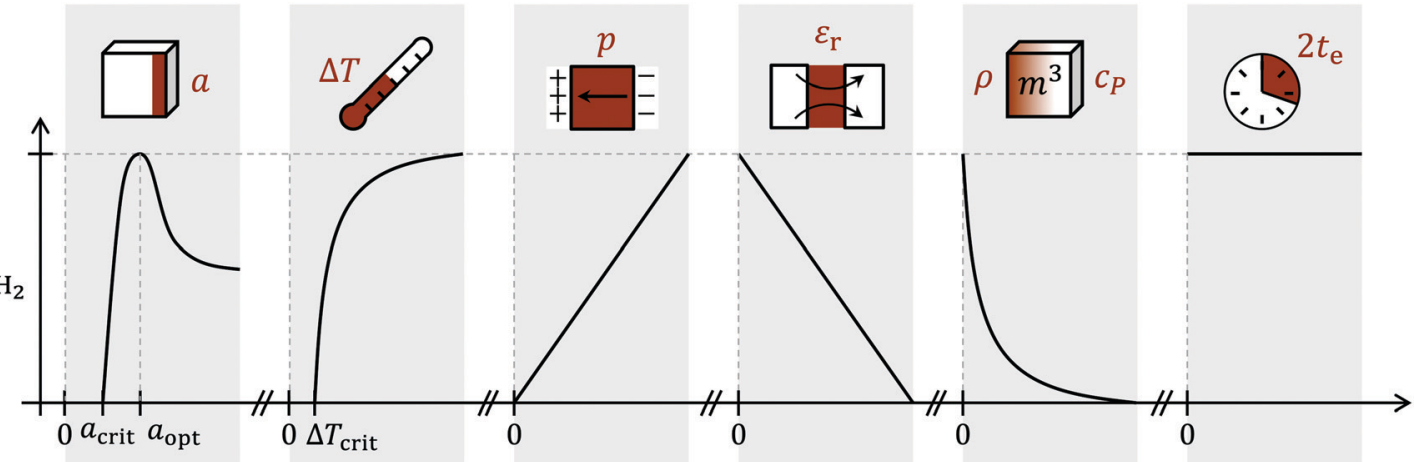

(b) $\mathrm{H}_{2}$

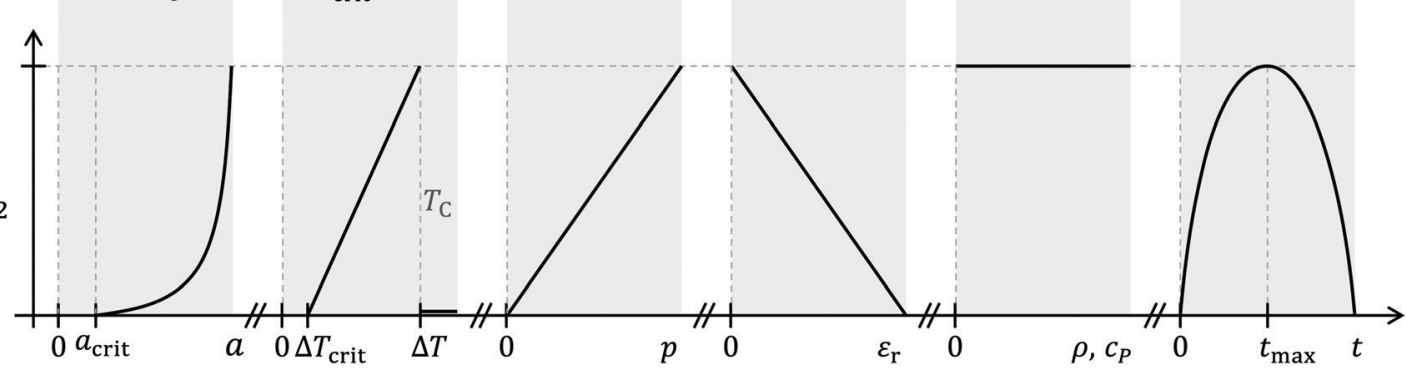

Fig. 6 Influence of particle size $a$, temperature difference $\Delta T$, pyroelectric coefficient $p$, relative permittivity $\varepsilon_{r}$, density $\rho$, heat capacity $c_{p}$ and cycle duration $2 t_{\mathrm{e}}$ on (a) the efficiency of the chemical conversion $\eta_{\mathrm{H}_{2}}$ and (b) hydrogen generation per time. Schematic representation based on eqn (24) (a) and eqn (21) (b). Both equations are time-independent. However, high cycling times may lead to non-selective oxidation/corona discharge while low cycling times may be screened by sheer electrostatics (capacitive regime), thus reducing the hydrogen output. ${ }^{25}$ For a high $\mathrm{H}_{2}$ generation output, $\Delta T$ and $p$ should be maximised, while keeping $\varepsilon_{r}, \rho$ and $c_{p}$ low.

another, so that it is difficult to increase $p$ while keeping $\varepsilon_{\mathrm{r}}$ small. $^{42}$

$$
\eta_{\mathrm{H}_{2}, \max }=\frac{\eta_{\mathrm{F}} V_{\mathrm{rx}} \bar{p}^{2} \Delta T}{8 \rho \bar{c}_{\mathrm{P}} V_{\mathrm{th}} \varepsilon_{0} \bar{\varepsilon}_{\mathrm{r}}}
$$

Temperature also plays a crucial role in the generation of the threshold potential. From eqn (24) it is possible to calculate the minimum temperature difference necessary for reaction by equating $Q_{\text {tot }}$ to $Q_{\text {th }}$ (eqn (29)).

$$
\Delta T_{\text {crit }}=\frac{2 V_{\text {th }} \varepsilon_{0} \bar{\varepsilon}_{\mathrm{r}}}{\bar{p} a}
$$

In addition, it would be advantageous to minimise $c_{\mathrm{P}}$, as the same chemical conversion could be achieved with a reduced heat input $q_{\text {in }}$. That would allow for quicker temperature cycling, too. Fig. 6 schematically shows the roles of the aforementioned parameters on the hydrogen yield.

\subsection{Figure of merit}

Upon examination of eqn (28) we encounter four types of quantities: constants (such as $\left.\varepsilon_{0}\right)$, pseudo-constants (e.g. $\eta_{\mathrm{F}}$ or $V_{\text {rx }}$ ), process-related factors (such as $\Delta T$ ) and the material properties $\rho, p(T), c_{\mathrm{P}}, \varepsilon_{\mathrm{r}}$ and $V_{\mathrm{th}}$. When it comes to comparing different pyroelectric materials, the pseudo-constants will be determined by the experimental setup used - that is the reaction of interest, reactor characteristics, etc. We propose a figure of merit (FoM) for the PTC based on eqn (28), which depends on these parameters as well as on $\eta_{\mathrm{op}}$ in order to account for the reaction of interest and band gap.

$$
\mathrm{FoM}=\frac{\bar{p}^{2} \Delta T V_{\mathrm{rx}}}{\rho \bar{c}_{\mathrm{P}} \varepsilon_{0} \bar{\varepsilon}_{\mathrm{r}} V_{\mathrm{th}}}
$$

\section{Model calculations}

The PTC brings new efficiency terms into the yield calculation. Instead of directly converting the charge $Q_{\text {tot }}$ found in eqn (12) to the quantity of hydrogen moles - through multiplication with the number of particles $N_{\text {particles}}$, division by the Faraday constant and the number of stoichiometric electrons $\left(n_{\mathrm{e}}-\left(\mathrm{H}_{2}\right)=2\right.$, see eqn (3)) - the charge is first multiplied with $\eta_{\text {chem }}$. The corrected charge $Q_{\mathrm{rx}}$ is the one that relates to hydrogen.

We set up the model on Python ${ }^{43}$ and calculated the outputs for all three hydrogen experiments reported in literature. The temperature excitation was assumed to be sinusoidal, as that shown in Fig. 4. The results are shown in Table 2. Eqn (12) represents the output without the PTC efficiency correction $\eta_{\text {chem }}$. The comparison between the columns experiment - retrieving the authors measurements - and PTC - showing the model outputs - reveals very good correlations in two of three cases. For black phosphorene, a unique pyroelectric in terms of a large pyroelectric coefficient with a tiny relative permittivity, a large amount of charge is displaced with temperature, so that it reaches a thermal-tochemical conversion efficiency of $\eta_{\mathrm{H}_{2}}=0.228$ (refer to the ESI $\dagger$ ). The model predicts the hydrogen outcome very accurately.

For $\mathrm{BaTiO}_{3}$, the model predicts about one order of magnitude more than reported. The authors of this study provided neither 
Table 2 Comparison of experimental and modelled hydrogen yields normalised to grams of pyroelectric material and cycle

\begin{tabular}{|c|c|c|c|c|c|c|c|c|}
\hline Material & $\Delta T(\mathrm{~K})$ & Experiment $\left(\mathrm{mol} \mathrm{g}^{-1}\right)$ & PTC $\left(\mathrm{mol} \mathrm{g}^{-1}\right)$ & Eqn (12) $\left(\mathrm{mol} \mathrm{g}^{-1}\right)$ & $\eta_{\text {chem }}(-)$ & $E_{\mathrm{g}}{ }^{\prime}(\mathrm{eV})$ & FoM $(-)$ & $\eta_{\mathrm{H}_{2}}$ \\
\hline Black phosphorene $^{16}$ & 50 & $2.25 \times 10^{-5}$ & $2.49 \times 10^{-5}$ & $3.35 \times 10^{-3}$ & 0.0074 & 0.6 & $2.09 \times 10^{1}$ & $2.28 \times 10^{-1}$ \\
\hline $\mathrm{BaTiO}_{3}^{44}$ & 30 & $2 \times 10^{-12}$ & $29 \times 10^{-12}$ & $110 \times 10^{-12}$ & 0.275 & 3.08 & $4.29 \times 10^{-5}$ & $1.52 \times 10^{-6}$ \\
\hline $\mathrm{Ba}_{0.7} \mathrm{Sr}_{0.3} \mathrm{TiO}_{3}{ }^{31}$ & 25 & $1.30 \times 10^{-6}$ & 0 & $3.36 \times 10^{-7}$ & 0 & 0.25 & $3.04 \times 10^{-4}$ & 0 \\
\hline
\end{tabular}

blank measurements nor exactly quantifiable figures on reactor volume and number of cycles, so that it is not meaningful trying to improve the accuracy further. The following model parameters were employed: $\eta_{\mathrm{F}}=1$ (experiments will be likely to have a lower value), a particle size $a=200 \mathrm{~nm}$ (the particle size distribution reported was neglected). The predicted outcomes still represent an improvement of roughly one order of magnitude in comparison to eqn (12).

The case of $\mathrm{Ba}_{0.7} \mathrm{Sr}_{0.3} \mathrm{TiO}_{3}$ is different. Due to the very large value of $\bar{\varepsilon}_{\mathrm{r}}$, the displaced charge is far from producing the electric potential $V_{\mathrm{th}}$, required for reactions. The PTC expresses this insufficiency in form of a $\Delta T_{\text {crit }}=498 \mathrm{~K}$ (see Table S1, ESI $\dagger$ ) for the given particle size or alternatively a critical particle size $a_{\text {crit }}=3.98 \times 10^{-6} \mathrm{~m}$ for the temperature difference applied. Both parameters are 20 times larger than their experimental counterparts. Accordingly, the model predicts an outcome of $0 \mathrm{~mol} \mathrm{H}_{2}$. We believe the experiment might have been affected by some level of interference with sonolytically produced radicals or with simultaneous piezoelectric phenomena (all pyroelectrics are piezoelectric). The PTC model is concerned only with pyroelectrocatalysis.

Essential parameters for the simulation are particle size and band gap. The small $\eta_{\text {chem }}$ value for black phosphorene is a consequence of the very thin material layer used, which is just above the critical thickness for the creation of the threshold voltage. You et al. ${ }^{16}$ report a band gap narrowing for increasing number of phosphorene layers. The band gap varies between $1.8 \mathrm{eV}$ in the monolayer and $0.3 \mathrm{eV}$ in the bulk, being around $0.7 \mathrm{eV}$ for a few layers. Tables S1 and S2 in the ESI $\dagger$ present all calculated values as well as the influence of the surface band gap on the outputs. To generalise, the broader $E_{\mathrm{g}}{ }^{\prime}$, the more energy is used for reaching the threshold voltage, the less charge is available for chemistry.

\section{Conclusion}

Pyroelectrocatalysis is the process of converting thermal energy directly into chemical energy with a pyroelectric material. The pyroelectric must be in contact with the chemically labile medium (here water) and chemical reactions occur by transferring charges into and out of the reactive medium.

This article introduces a new thermodynamic cycle, termed pyroelectrocatalytic threshold cycle, PTC, which models pyroelectrocatalysis more accurately. Its main characteristic is the need for a minimum threshold potential $V_{\text {th }}$ which depends on the chemical reaction of interest on the one hand and the band structure of the pyroelectric semiconductor - and its surface on the other hand. Before this potential is built up, no chemical conversion can take place and the thermal energy is lost. After the potential reaches $V_{\text {th }}$, it does not increase further because the emerging surface-bound charges are compensated through charge transfer with the fluid. The potential remains constant until the next event begins.

The temperature difference and the particle size determine whether enough charge can accumulate for reaction. Smaller particles invest comparatively more energy in reaching the threshold and leave less room for reactions. At the same time they possess more active area, so that a trade-off between charge efficiency and active site utilisation arises.

Reported experimental hydrogen yields could be modelled with the PTC to an accuracy of about one order of magnitude. The current yields correspond to conversion efficiencies in the range $10^{-1}-10^{-6}$, even for small temperature differences. This waste-heat utilisation potential should encourage further studies on the physical and chemical limits of pyroelectric materials.

\section{Conflicts of interest}

There are no conflicts to declare.

\section{Acknowledgements}

We dedicate this article to Prof. Peter Paufler (TU Dresden) as mentor of crystal physics. This work was initially performed within the junior research group PyroConvert (100109976, 100152607) financially supported by the Ministry of Science and Art of Saxony (SMWK), the Sächsische Aufbaubank (SAB) and the European Union (ESF, EFRE). Further research was performed within the collaborative research projects CryPhysConcept (03EK3029A) and R2RBattery (03SF0542A) funded by the German Federal Ministry of Education and Research (BMBF). We are grateful to Ms Kerstin Annassi from Project Management Jülich for her professional assistance in these projects, thanks to whom the results included in this publication have been achieved.

\section{Notes and references}

1 C. Forman, I. K. Muritala, R. Pardemann and B. Meyer, Renewable Sustainable Energy Rev., 2016, 57, 1568-1579.

2 M. Pehnt, J. Bodeker, M. Arens, E. Jochem and F. Idrissova, Proceedings of ECEEE, 2011.

3 U.S. Department of Energy, Waste Heat Recovery: Technology and Opportunities in U.S. Industry, 2008.

4 European Commission, 100 Radical Innovation Breakthroughs for the future, 2019.

5 S. B. Lang, Sourcebook of Pyroelectricity, Gordon and Breach, Science Publishers, Inc., New York, 1974, vol. 2.

6 S. Jachalke, E. Mehner, H. Stöcker, J. Hanzig, M. Sonntag, T. Weigel, T. Leisegang and D. C. Meyer, Appl. Phys. Rev., 2017, 4, 021303. 
7 P. Paufler, Physikalische Kristallographie, Akademie-Verlag Berlin, 1986, p. 325.

8 R. E. Newnham, Properties of Materials: Anisotropy, Symmetry, Structure, Oxford University Press, 1st edn, 2005.

9 W. Kleber, Einführung in die Kristallographie, De Gruyter Oldenbourg, 19th edn, 2010.

10 Q. Leng, L. Chen, H. Guo, J. Liu, G. Liu, C. Hu and Y. Xi, J. Mater. Chem. A, 2014, 2, 11940-11947.

11 F. Y. Lee, A. Navid and L. Pilon, Appl. Therm. Eng., 2012, 37, 30-37.

12 S. K. T. Ravindran, M. Kroener and P. Woias, Procedia Eng., 2012, 47, 33-36.

13 S. Krishnan, D. Ezhilarasi, G. Uma and M. Umapathy, IEEE Trans. Sustain. Energy, 2014, 5, 73-81.

14 E. Gutmann, A. Benke, K. Gerth, H. Böttcher, E. Mehner, C. Klein, U. Krause-Buchholz, U. Bergmann, W. Pompe and D. C. Meyer, J. Phys. Chem. C, 2012, 116, 5383-5393.

15 J. Wu, W. Mao, Z. Wu, X. Xu, H. You, A. Xue and Y. Jia, Nanoscale, 2016, 8, 7343-7350.

16 H. You, Y. Jia, Z. Wu, F. Wang, H. Huang and Y. Wang, Nat. Commun., 2018, 9, 2889.

17 A. Benke, E. Mehner, M. Rosenkranz, E. Dmitrieva, T. Leisegang, H. Stöcker, W. Pompe and D. C. Meyer, J. Phys. Chem. C, 2015, 119, 18278-18286.

18 A. Kakekhani and S. Ismail-Beigi, J. Mater. Chem. A, 2016, 4, 5235-5246.

19 M. Xie, S. Dunn, E. L. Boulbar and C. R. Bowen, Int. J. Hydrogen Energy, 2017, 42, 23437-23445.

20 Y. Zhang, S. Kumar, F. Marken, M. Krasny, E. Roake, S. Eslava, S. Dunn, E. Da Como and C. R. Bowen, Nano Energy, 2019, 58, 183-191.

21 R. B. Olsen, D. A. Bruno and J. M. Briscoe, J. Appl. Phys., 1985, 58, 4709-4716.

22 C. Bowen, J. Taylor, E. LeBoulbar, D. Zabek, A. Chauhan and R. Vaish, Energy Environ. Sci., 2014, 7, 3836-3856.

23 E. Lefeuvre, A. Badel, C. Richard and D. Guyomar, J. Intell. Mater. Syst. Struct., 2005, 16, 865-876.

24 G. Sebald, E. Lefeuvre and D. Guyomar, IEEE Trans. Ultrason. Ferroelectr. Freq. Control, 2008, 55, 538-551.
25 J. Schlechtweg, S. Raufeisen, M. Stelter and P. Braeutigam, Phys. Chem. Chem. Phys., 2019, 21, 23009-23016.

26 M. B. Starr and X. Wang, Sci. Rep., 2013, 3, 1-8.

27 C. Grimes, O. Varghese and S. Ranjan, in Light, Water, Hydrogen, ed. C. Grimes, O. Varghese and S. Ranjan, Springer US, 2008, pp. 35-113.

28 V. E. Henrich, The Surface Science of Metal Oxides, Cambridge Univ. Press, 2000.

29 V. E. Henrich, The Chemical Physics of Solid Surfaces, in The Chemical Physics of Solid Surfaces, ed. D. Woodruff, Elsevier, 2001, vol. 9, pp. 1-34.

30 R. L. LeRoy, C. T. Bowen and D. J. LeRoy, J. Electrochem. Soc., 1980, 127, 1954-1962.

31 X. Xu, L. Xiao, Y. Jia, Z. Wu, F. Wang, Y. Wang, N. O. Haugen and H. Huang, Energy Environ. Sci., 2018, 11, 2198-2207.

32 T. Perls, T. Diesel and W. Dobrov, J. Appl. Phys., 1958, 29, 1297-1302.

33 S. H. Wemple, Phys. Rev. B: Solid State, 1970, 2, 2679-2689.

34 M. Srinivasan, Bull. Mater. Sci., 1984, 6, 317-325.

35 S. Kase and K. Ohi, Ferroelectrics, 1974, 8, 419-420.

36 H. P. Beerman, Infrared Phys., 1975, 15, 225-231.

37 R. B. Maciolek and S. T. Liu, J. Electron. Mater., 1973, 2, 191-200.

38 F.-P. Koffyberg, K. Dwight and A. Wold, Solid State Commun., 1979, 30, 433-437.

39 M. Durruthy-Rodríguez, J. Costa-Marrero, M. HernándezGarcía, F. Calderón-Piñar and J. Yañez-Limón, Appl. Phys. A: Mater. Sci. Process., 2010, 98, 543-550.

40 M. Olszowy, E. Nogas-Ćwikiel and K. Ćwikiel, J. Phys.: Conf. Ser., 2011, 289, 012017.

41 Y. Tang, X. Wan, X. Zhao, X. Pan, D. Lin, H. Luo, J. Sun, X. Meng and J. Zhu, J. Appl. Phys., 2005, 98, 0841041.

42 S. T. Liu, J. D. Zook and D. Long, Ferroelectrics, 1975, 9, 39-43.

43 G. van Rossum, Python tutorial, Centrum voor Wiskunde en Informatica (CWI) Technical Report CS-R9526, 1995.

44 R. Belitz, P. Meisner, M. Coeler, U. Wunderwald, J. Friedrich, J. Zosel, M. Schelter, S. Jachalke and E. Mehner, Energy Harvest. Syst., 2017, 4, 107-113. 